\title{
A Distributed Coordination Framework for Adaptive Sensor Uncertainty Handling
}

\author{
Zhifeng Dai ${ }^{1}$, Yuanxiang $\mathrm{Li}^{2}$, Bojin Zheng ${ }^{3}$, and Xianjun Shen ${ }^{4}$ \\ ${ }^{1}$ School of Computer, Wuhan University \\ ${ }^{2}$ State Key Lab of Software Engineering, Wuhan University \\ ${ }^{3}$ College of Computer Science, South-Central University for Nationalities \\ ${ }^{4}$ Department of Computer Science, Huazhong Normal University \\ 430072 Wuhan, China \\ dwjse@163.com, yxli@whu.edu.cn, zhengbojin@gmail.com
}

\begin{abstract}
The evaluation and management of sensor uncertainty is particularly necessary in a noisy multi-sensor context. In this paper, focusing on the potential of distributed coordination among sensor nodes based on the built-in association between wireless sensor networks and multi-agent systems, meanwhile in a rough set technique sense of uncertainty, we show how an adaptive distributed coordination framework for a hierarchy of sensor data uncertainty performs local data fusion to increase the certainty of real-time sensor readings coherently, makes global rational decisions under imprecision and partial truth, and reconciles the conflicts somewhat, thus evolving adaptive and robust sensor uncertainty handling systems. Implementation results for an example sensor field demonstrate the application of our proposed approach.
\end{abstract}

Keywords: adaptive distributed coordination strategy, rough set theory, multiagent systems, modeling sensor uncertainty, wireless sensor networks.

\section{Introduction}

Generally, an explicit definition of uncertainty may refer to the idea of an information gap between what we do know and what we need to know [1]. However, as a suitable mathematical formalization of uncertainty, rough set theory can quantify both what is known and what is unknown. Moreover, from rough set perspective, further research is needed to view the ways in which uncertainties are hierarchical and interconnected. Especially, so far there exists little work on incorporating coordination mechanism into adaptive sensor uncertainty handling, for which the issue of a distributed coordination framework is actually an important and novel research direction.

\section{Background Concepts and Motivation}

Rough set theory approaches the problem of inexact concepts in the form of a decision system $(U, A \cup\{d\})$. Here $U$ is a non-empty finite set of objects, $A$ is a set of condition attributes, and $d$ is decision element. For any subset $R$ of $A, A S=(U, R)$, 
an ordered pair, is the approximation space [2]; $[\mathrm{x}]_{\mathrm{R}}$ denotes the equivalence class of $\mathrm{R}$ containing $\mathrm{x}$, for $\mathrm{x} \in \mathrm{U}$. The lower approximation of $\mathrm{X}$ is the set $\underline{R} X=\{\mathrm{x} \mid \mathrm{x} \in \mathrm{U}$, $\left.[\mathrm{x}]_{\mathrm{R}} \subseteq \mathrm{X}\right\}$ and the upper approximation of $\mathrm{X}$ is the set $\bar{R} X=\left\{\mathrm{x} \mid \mathrm{x} \in \mathrm{U},[\mathrm{x}]_{\mathrm{R}} \cap \mathrm{X} \neq \varnothing\right\}$. The results in $\underline{R} X$ are certain. The boundary region $\bar{R} X-\underline{R} X$ contains those uncertainty results that are possible, but not certain.

Multi-agent systems are fundamentally designed for dealing with and offering architecture for complex distributed applications that require collaborative problem solving, and wireless sensor networks consist of a collection of light-weight sensors connected via wireless links to each other or to a more powerful gateway node [3].

While principles taken from the rough set theory are suitably employed for the mathematical modeling and further handling of sensor uncertainty through the use of its approximation space, the need for cooperative processing of spatially and temporally dense collected data because of limited resources, makes multi-agent system architecture an ideal candidate for analyzing and modeling distributed sensor data, and these ideas are the motivation behind this approach.

\section{Modeling of Sensor Uncertainty}

Herein, we identify uncertainty as a measure of the level of confidence of a sensed value, or of validity of a conclusion at a higher level. Due to hardware limitations and signal processing inaccuracies, there are always some forms of uncertainty associated with sensor measurement and the processed information. First, some uncertainties can only be inferred as the absence or incompleteness of certain observational data item values because of inaccessibility. And second, uncertainty can be seen as a scaling of the noise level of inconsistent data sets, also in the form of the binary data inconsistency failures during the actual sensor processing. Additionally, a sensor node usually faces uncertainties owing to its limited or outdated partial information about the states of complex problem solving in other nodes. Furthermore, there exists the uncertainty which arises from the issue of granularity effect. All these form a hierarchy of sensor data uncertainty.

\section{Adaptive Distributed Sensor Uncertainty Coordination Frame}

In order to effectively handle sensor uncertainty, as shown in Figure 1, we may use the term "agent" to mean any sensor node, and there are three kinds of hierarchically dispersed agents embedded on sensors, i.e. sensor agents, cluster agents, and the manager agent, which form a wireless sensor network into a distributed multi-agent sensor system. Furthermore, as coordination is the process of effectively managing interdependencies between activities distributed across agents [4], we propose a hierarchical distributed coordination framework for sensor uncertainty handling, which bridges the potential of adaptive coordination to reduce sensor uncertainty with that of intelligent decision in the form of rule with an appropriate level of certainty. 


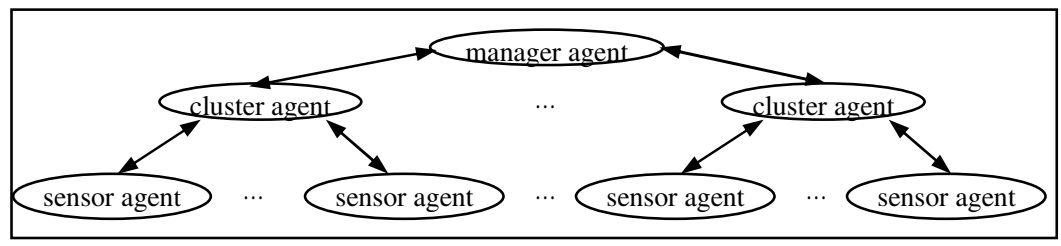

Fig. 1. Distributed multi-agent wireless sensor networks

Firstly, on the layer of local data fusion, the principle issue is to manage and minimize the measured sensor agent uncertainties through suitable fusion algorithms. On the one hand, redundant data fusion can increase the accuracy and certainty of sensed data in the case of sensor failure. On the other hand, complementary data fusion allows features to be sensed coherently through fusing the different types of data from more than one source sensors to synthesize meaningful information.

Secondly, on the layer of attribute reduction and decision rule, the major topics consist in the fact that one must not only be aware of sensor uncertainty, but also discover hidden concise relationships that agree with uncertain sensor situations. At the cluster agents, by employing the notion of reduction in a rough set sense, which is the minimal set of condition attributes that eliminates pairwise dependencies and makes the same decisions as the whole set, those redundant condition attributes along with their associated uncertainties may be removed, then the reduction of the number of attributes and further the discovery of deterministic decision rules are achieved, thus providing an appropriate model for the distributed multi-agent sensor system.

Thirdly, on the layer of global conflict resolution at the manager agent, problem of conflict resolution is of most importance due to exclusive interpretations of the same thing or conflicting sets of rules from different source sensors. In rough set approach, inconsistency is represented by the concepts of lower and upper approximation, and confliction relation is expressed by the notion of boundary region, which may be of minimal level of uncertainty by forcing a finer granulation of the partitioning. Moreover, through the use of explicit rule priorities, we can reconcile the consistency degrees about the inferences under uncertainty as far as possible.

\section{Case Study: Example Implementation Results}

In this section we illustrate the above presented ideas by a distributed multi-sensor paradigm for real-time forest fire detection. Herein, the sensor data can be represented as a decision table, which is constitutive of condition attributes \{temperature, relative humidity, rainfall, smoke, wind speed\} and decision attribute \{fire level\}.

Here we discuss some primary kinds of hierarchical sensor uncertainty at the cluster agents and the manager agent. A schedule of these uncertainties and the corresponding example implementation results are listed in Table 1 and Table 2, respectively. While raw uncertainty sensor data from distributed sensor agents and cluster agents have been converted into the minimal efficient sets of certain condition attribute data and deterministic decision rules at the manager agent, these demonstrate the efficiency of the adaptive distributed sensor uncertainty coordinate framework. 
Table 1. A schedule of hierarchical sensor uncertainties

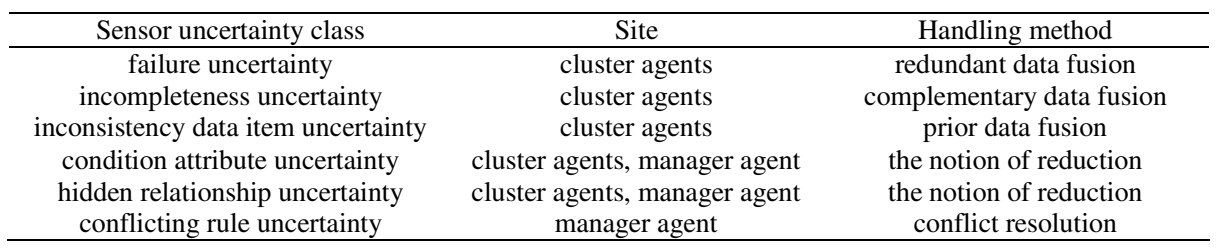

Table 2. Summary of kinds of uncertainty handling in distributed multi-agent sensor system

\begin{tabular}{|c|c|c|c|c|c|c|c|c|c|c|c|c|}
\hline \multirow[t]{2}{*}{$\begin{array}{l}\text { Agent } \\
\text { class }\end{array}$} & \multirow[t]{2}{*}{$\begin{array}{l}\text { Node } \\
\text { num }\end{array}$} & \multicolumn{2}{|c|}{$\begin{array}{c}\text { Failure } \\
\text { uncertainty }\end{array}$} & \multicolumn{2}{|c|}{$\begin{array}{c}\text { Incompleteness } \\
\text { uncertainty }\end{array}$} & \multicolumn{2}{|c|}{$\begin{array}{c}\text { Inconsistency } \\
\text { data item } \\
\text { uncertainty }\end{array}$} & \multicolumn{2}{|c|}{$\begin{array}{l}\text { Condition } \\
\text { attribute } \\
\text { uncertainty }\end{array}$} & \multirow[t]{2}{*}{$\begin{array}{l}\text { Hidden } \\
\text { relationship } \\
\text { uncertainty }\end{array}$} & \multicolumn{2}{|c|}{$\begin{array}{c}\text { Conflicting } \\
\text { rule } \\
\text { uncertainty } \\
\end{array}$} \\
\hline & & total & disposal & total & disposal & total & disposal & total & 1 reduction & & total & disposal \\
\hline cluster1 & 40 & 4 & 4 & 12 & 12 & 4 & 4 & 5 & 2 & 3 & 0 & 0 \\
\hline & 50 & 6 & 6 & 2 & & 8 & & 5 & & & 0 & 0 \\
\hline & 36 & 4 & 4 & 10 & 10 & 2 & & 5 & & 3 & 0 & 0 \\
\hline cluster4 & 52 & 8 & 8 & 24 & 24 & 6 & 4 & $J$ & 2 & 3 & 0 & 0 \\
\hline manager & c 178 & 0 & 0 & 0 & 0 & 0 & 0 & 5 & 2 & 3 & 2 & 2 \\
\hline
\end{tabular}

\section{Conclusions}

Considering there is some room for a mixture of approaches for the sensor uncertainty problem based on rough set theory and multi-agent system, we have proposed and demonstrated an adaptive distributed coordination frame reflecting the handling of multiple levels of sensor uncertainty, along with emphasis on how to make a complex multi-agent sensor system self-repairing and behave deterministically to some extent.

Acknowledgments. The authors would like to thank the National Science Foundation of P.R.C for financial support under Grant No. 60473014 for this research.

\section{References}

1. Hatfield A. J., Hipel K W.: Understanding and Managing Uncertainty and Information. IEEE International Conference on Systems, Man, and Cybernetics, Vol. 5. IEEE Computer Society (1999) 1007-1012

2. Theresa Beaubouef, Frederick Petry: Vague Regions and Spatial Relationships: A Rough Set Approach. Proceedings of the Fourth International Conference on Computational Intelligence and Multimedia Applications. IEEE Computer Society (2001) 313-317

3. Chris Giannella, Ruchita Bhargava, Hillol Kargupta: Multi-agent Systems and Distributed Data Mining. In: Klusch M. et al. (eds.): CIA 2004, LNAI 3191. Springer- Verlag (2004) 1-15

4. Nagendra Prasad M. V., Lesser Victor R.: Learning Situation-Specific Coordination in Cooperative Multi-agent Systems. Autonomous Agents and Multi-agent Systems, 2. Kluwer Academic (1999) 173-207 